\title{
On the Effective Thermal Conductivity of Porous Packed Beds with Uniform Spherical Particles
}

\author{
M. Kandula \\ ASRC Aerospace, John F. Kennedy Space Center, FL, USA \\ E-mail address: Max.Kandula-1@ksc.nasa.gov
}

\begin{abstract}
Point contact models for the effective thermal conductivity of porous media with uniform spherical inclusions have been briefly reviewed. The model of Zehner and Schlunder (1970) has been further validated with recent experimental data over a broad range of conductivity ratio from 8 to 1200 and over a range of solids fraction up to about 0.8 . The comparisons further confirm the validity of Zehner-Schlunder model, known to be applicable for conductivity ratios less than about 2000, above which area contact between the particles becomes significant. This validation of the Zehner-Schlunder model has implications for its use in the prediction of the effective thermal conductivity of water frost (with conductivity ratio around 100) which arises in many important areas of technology.
\end{abstract}

Key Words: Zehner-Schlunder model, spherical packed bed, effective conductivity.

\section{Nomenclature}

$B=$ particle shape factor

$k=$ thermal conductivity

$k_{e}=$ effective thermal conductivity of porous medium

\section{Greek Symbols}

$\phi=$ solids fraction

$\psi=$ porosity (void fraction)

$\lambda=$ solid-to-fluid thermal conductivity ratio, $k_{2} / k_{1}$

$\rho=$ density 
$\xi=$ fluid-to-solid thermal conductivity ratio, $k_{1} / k_{2}$

\author{
Subscripts \\ $e=$ effective medium \\ $1=$ continuous medium (solid or fluid) \\ $2=$ solid particle (dispersed)
}

\title{
1. Introduction
}

Heat transport through porous media is of great interest in chemical, mechanical, geological, environmental and petroleum applications (Kaviany, 1995). Packed beds are widely used in various industrial equipment including heat exchangers, dryers, absorbers, distillation and extraction columns, chemical reactors, calcinators, and incinerators. Porous media applications arise also in the design of cryocoolers, heat pipes, enhanced oil recovery, and geothermal and petroleum applications (involving porous rocks and soil). Recent applications of porous media arise in microelectronics for electronic packaging (device encapsulation) as thermal interface materials (TIM) for efficient disposal of the generated heat. Composites in the form of porous media tend to manifest in greatly improved physical properties. The determination of the effective thermal conductivity of various porous media such as granular materials (randomly packed), fibrous composites, and packed beds is of great practical interest in the eefcient design of industrial equipment.

The structure of a porous medium is very complex, consisting of different grain (pore) sizes and geometries (Cheng and Hsu, 1999). A detailed prediction of the effective thermal conductivity of heterogeneous media requires a knowledge of the shape, size, location (distribution) and conductivity of each particle in the system together with interaction between particles (Crane \& Vachon, 1977). For randomly packed systems, such information is difficult to represent. On account of the various possible packing arrangements, for given phases the effective thermal conductivity is not a unique function of solids fraction, but a band of effective thermal conductivity for the packed beds (of microspheres and fibrous insulation media) is shown to exist with the aid of statistical considerations (Tien and Vafai, 1979).

Many models are based on simple geometries with periodic structure. Broadly speaking, there are two basic approaches considered with simplifying assumptions. In the Fourier's law models, simplified geometry is considered with solution of the Laplace equation. In Ohm's law (electrical network) models, a one-dimensional heat flow assumption is considered. A comprehensive review of empirical correlations for thermal conductivity of porous media was given by Kaviany (1995), see Feng (2004). 
Numerous models for the effective thermal conductivity of a medium made up from inclusions (spheres, cylinders, irregular particles) of one substance embedded in a matrix of a different material has been investigated since the classical work of Maxwell (1865). Maxwell's model, applicable to very dilute suspensions, has been extended by Lord Rayleigh (1892) and many others for spherical as well as cylindrical particles with point contact between the particles. These models were limited to solids fraction $\phi$ less than 0.5236 for spherical inclusions with simple cubic packing (and less than 0.7854 for cylindrical inclusions). Note that The maximum value of solid fraction that is possible in a packed bed of uniform spherical particles is 0.524 for simple cubic packing, 0.68 for body-centered cubic, and 0.74 for face-centered packing (Tien and Vafai 1979).

Among the point contact models, Zehner and Schlunder's model (1970), applicable to spheres, cylinders and irregular (rough) particles over the entire range of solid fractions, has been tested against the data of Nozad et al. (1985) for $\phi \approx 0.6$ shown to be satisfactory over a wide range of solid-to-fluid conductivity ratio $k_{2} / k_{1}$ less than 2000 (Kaviany 1995; Cheng and Hsu, 1999). For $k_{2} / k_{1}>2000$, the data considerably exceed the prediction of Zhner-Schlunder model. It has been shown that (Hsu et al., 1994) the area contact becomes important in that range, and accordingly modified the Zehner-Schlunder model by accounting for area contact heat transfer. In this reference, comparisons of the Zehner-Schlunder model with data was considered at a single value of $\phi \approx 0.6$.

One important application of Zehner and Schlunder model (1970) is in the determination of the effective thermal conductivity of frost, with a solid-to-fluid conductivity ratio about 100 . Frost is generally undesirable in industrial applications (except in freeze-drying of food), and arises in cryocoolers, refrigeration and air conditioning equipment, heat exchangers, and liquid propellant storage and transportation (aerospace). It is the purpose of this work to validate Zehner-Schlunder model over a broad range of $k_{2} / k_{1}$ below 2000 and a range of solids fraction by comparing the model to more recent experimental data, so that the model's usefulness for frost and related applications can be considered.

\section{Physical Models}

\subsection{Limiting Models}

Two limiting cases are provided by the series distribution (where the two phases are thermally in series with respect to the direction of heat flow), and the parallel distribution (in which the two phases are thermally in parallel with respect to the direction of heat flow), see Meredith and Tobias (1960). The effective medium thermal conductivity $k_{e}$ under these circumstances is expressed by

$$
\frac{k_{e}}{k_{1}}=\frac{k_{2} / k_{1}}{(1-\psi)+\psi\left(k_{2} / k_{1}\right)}=\frac{k_{2} / k_{1}}{\phi+(1-\phi)\left(k_{2} / k_{1}\right)} \quad \text { minimum(series) }
$$




$$
\frac{k_{e}}{k_{1}}=\psi+(1-\psi) \frac{k_{2}}{k_{1}}=(1-\phi)+\phi \frac{k_{2}}{k_{1}} \quad \operatorname{maximum}(\text { parallel })
$$

where the porosity $\psi$ is related to the solids fraction by the relation

$$
\psi=1-\phi
$$

The solids fraction can be expressed in terms of the densities of the constituent phases $\rho_{1}, \rho_{2}$ as

$$
\phi=\left(\rho-\rho_{1}\right) /\left(\rho_{2}-\rho_{1}\right)
$$

so that

$$
\psi=\left(\rho_{2}-\rho\right) /\left(\rho_{2}-\rho_{1}\right)
$$

where $\rho_{1}$ refers to the continuous phase, and $\rho_{2}$ refers to the dispersed (solid) phase.

\subsection{Maxwell's Model}

For a sufficiently dilute dispersion (suspension) of spheres and taking into account only the induced dipole moments of the spherical particles in a temperature field, Maxwell (1865) obtained the following expression for the stagnant thermal conductivity of a packed-sphere bed:

$$
\begin{aligned}
\frac{k_{e}}{k_{1}} & =\frac{2 \psi+\left(k_{2} / k_{1}\right)(3-2 \psi)}{3-\psi+\left(k_{2} / k_{1}\right) \psi} \\
& =1+\frac{3\left(k_{2} / k_{1}-1\right) \phi}{\left(1-k_{2} / k_{1}\right) \phi+\left(k_{2} / k_{1}+2\right)}=\frac{\lambda+2-2 \phi(1-\lambda)}{\lambda+2+\phi(1-\lambda)}
\end{aligned}
$$

where

$$
\lambda=k_{2} / k_{1}
$$

is the solid-to-fluid thermal conductivity ratio.

Maxwell's equation is valid for $\phi \rightarrow 0$, since it was derived on the assumption that the solid spheres are sufficiently apart that they do not mutually interact. It provides a lower bound for the stagnant thermal conductivity of a packed-sphere bed. This formula can also be used to calculate the dielectric constant, electrical conductivity, and magnetic susceptibility of composite materials.

Eucken (1932) generalized Maxwell's equation to the case of $n$ dispersed phases embedded in one continuous phase. Burger (1915) extended Maxwell's equation to ellipsoidal particles.

\subsection{Rayleigh's Model}


Lord Rayleigh (1892) extended Maxwell's model to higher order in the particle concentration $\phi$, by taking into account the concentration of induced octupole moments. The case of a cubic array of uniform size spheres (simple cubic packing) was considered, and the effect on the potential in the neighborhood of a sphere by 248 of its closest neighbors (lying within the first 15 shells around a central sphere) was investigated (Meredith \& Tobias, 1960). Rayleigh solved the Laplace equation for the potential inside and about a sphere invoking the principle of superposition to take into account the effect of surrounding spheres on the field in the neighborhood of the central sphere. The following expression is obtained for the effective thermal conductivity of the packed bed:

or

$$
\begin{aligned}
& \frac{k_{e}}{k_{1}}=\frac{[(2+\lambda) /(1-\lambda)]-2 \phi-0.525[(3-3 \lambda) /(4+3 \lambda)] \phi^{10 / 3}}{[(2+\lambda) /(1-\lambda)]+\phi-0.525[(3-3 \lambda) /(4+3 \lambda)] \phi^{10 / 3}} \\
& \frac{k_{e}}{k_{1}}=1-3 \phi\left[\frac{1+2 \lambda}{1-\lambda}+\phi-\frac{4.95(1-\lambda)}{(4+3 \lambda)} \phi^{10 / 3}+O\left(\phi^{14 / 3}\right)\right]^{-1}
\end{aligned}
$$

This expression approaches Maxwell's result by neglecting higher order terms in $\phi$ in Eq. (5a). In Rayleigh's result, Eq. (5a), a numerical correction was introduced by Runge (1925). The coefficient 0.525 replaces 1.65 in the denominator, correcting for the omission of a factor of $1 / \pi$ in Rayleigh's derivation (Meredith \& Tobias 1960).

\subsection{Extensions of Rayleigh's Model}

Several investigators extended Rayleigh's model to higher order. A review of these early non-touching models is given by Churchill (1986).

With increasing $\phi$, the discrepancy between Maxwell's result and Rayleigh's result become increasingly significant. At $\phi=0.5236$, the spherical particles in the cubic lattice are in point contact, and for $\lambda \rightarrow \infty$ the effective conductivity should approach $\infty$ at $\phi=0.5236$. These qualitative conditions are not satisfied by Rayleigh's expression (Meredith and Tobias, 1960).

By using a different function for the potential, and by considering higher order terms in the series expansion for the potential in the continuous phase, Meredith and Tobias (1960) modified Rayleigh's result, providing an analytical expression that agrees more satisfactorily with the data in the critical range near $\phi=0.5236$. This expression is as follows:

$$
\frac{k_{e}}{k_{1}}=\frac{\frac{2+\lambda}{1-\lambda}-2 \phi+0.409 \frac{6+3 \lambda}{4+3 \lambda} \phi^{7 / 3}-2.133 \frac{3-3 \phi}{4+3 \phi} \phi^{10 / 3}}{\frac{2+\lambda}{1-\lambda}+\phi+0.409 \frac{6+3 \lambda}{4+3 \lambda} \phi^{7 / 3}-0.906 \frac{3-3 \lambda}{4+3 \lambda} \phi^{10 / 3}}
$$


As $\lambda \rightarrow \infty$, the data are in satisfactory agreement with the modified expression, except for $\phi=0.5161$ where the data considerably exceed the theory (Meredith and Tobias, 1960).

\subsection{Model of Woodside}

Woodside (1961) considered a unit cube containing one-eighth of a sphere of radius $R$. The general case is considered in that the uniform spherical particles are not in contact. The final expression for the effective medium conductivity is shown to be

where

$$
\begin{gathered}
\frac{k_{e}}{k}=1-\left(\frac{6 \phi}{\pi}\right)^{1 / 3}\left[1-\left(\frac{a^{2}-1}{a}\right) \ln \left(\frac{a+1}{a-1}\right)\right] \\
a=\left[1+\frac{4}{\pi(\lambda-1)(6 \phi / \pi)^{2 / 3}}\right]^{1 / 2}
\end{gathered}
$$

In deriving the above equation, it was assumed that 1) the gas spaces are small enough that heat transfer by convection may be neglected, 2) the isotherms are planes perpendicular to the direction of heat flow. The second assumption is valid only when $k_{2} / k_{1} \approx 1$. The errors will be greater the larger the value of $k_{2} / k_{1}$.

As with Rayleigh's model, it is limited to $\phi=0.5236$. It appears that the accuracy of the model has not been sufficiently tested against measurements.

\subsection{Model of Zehner and Schlunder}

Zehner and Schlunder (1970) arrived at an analytical expression for the effective stagnant thermal conductivity of a packed bed. They considered for the unit cell one-eighth of a cylinder (inner cylinder of unit radius and outer cylinder of radius $R$ ). Fluid is filled between the inner and outer cylinders while the inner cylinder consists of both the solid and the fluid phases with its interface $A_{f s}$ described by (Cheng and Hsu, 1999)

$$
r^{2}+\frac{z^{2}}{[B-(B-1) z]^{2}}=1
$$

where $B$ is the shape factor characterizing the geometrical effect of the solid particle. For $B \rightarrow 0$ , the boundary becomes the $z$ axis with no solid volume; for $B=1$ the solid becomes a sphere, and for $B \rightarrow \infty$ the solid occupies the entire inner cylinder.

The effective thermal conductivity of a packed bed is expressed by 


$$
\begin{gathered}
\frac{k_{e}}{k_{f}}=1-\sqrt{1-\psi}+\frac{2 \sqrt{1-\psi}}{1-\xi B}\left[\frac{(1-\xi) B}{(1-\xi B)^{2}} \ln \left(\frac{1}{\xi B}\right)-\left(\frac{B+1}{2}\right)-\frac{B-1}{1-\xi B}\right] \\
\psi=1-\left[\frac{B\left(3-4 B+B^{2}+2 \ln B\right.}{(B-1)^{3}}\right]^{2}
\end{gathered}
$$

and

$$
\xi=k_{1} / k_{2}=1 / \lambda
$$

It was suggested that Eq. (10b) can be approximated by

$$
B=C\left(\frac{1-\psi}{\psi}\right)^{m}
$$

with $m=10 / 9$. The constant $C$ depends on the shape of the particle. Zehner and Schlunder (1970) suggest that $C=1.25,1.40$ and 2.5 for spheres, broken (irregular particles) and cylinders respectively.

A comparison of Zehner-Schlunder model with the data of Nozad et al. (1985), as mentioned in Cheng and Hsu (1999), suggests that the model with $\psi=0.4$ agrees well with the experimental data for $k_{2} / k_{1}<10^{3}$ (Fig. 1). For $k_{2} / k_{1}>10^{3}$, the Zehner-Schlunder model underpredicts the effective stagnant thermal conductivity substantially.

Hsu et al. (1995) postulated that the reason for this underprediction of data at large values of $k_{2} / k_{1}$ (high solid-to-fluid thermal conductivity ratio) is the assumption of point contacts between spheres. By considering the finite contact area between particles (arising from flattening of spheres due to external loads or the weight of the bed itself) in accordance with the Hertz relationship for elastic contacts (Hertz 1881; Timoshenko and Goodier 1970), they were able to obtain good agreement with data for $k_{2} / k_{1}$ up to about $10^{4}$. In addition to these so-called area contact models, Cheng (1999) discusses phase-symmetry models and lumped parameter models in this context.

\section{Comparisons}

Figure 2 shows a comparison of the model of Zehner and Schlunder (1970) with those of Maxwell (1865) along with the limiting models for a typical value of $k_{2} / k_{1}=100$. It is seen that the Zehner and Schlunder model always predicts higher effective conductivity than that of Maxwell for the entire range of the solid fractions $(0<\phi<1)$, while the Maxwell model exceed the minimum limit.

A comparison of Zehner-Schlunder model (1970) with other models (Maxwell, 1865; Rayleigh, 1892; Meredith and Tobias, 1915; Woodside, 1958) with the measurements of 
Kanuparthi et al. (2008) at $k_{2} / k_{1}=125$ is indicated in Fig. 3. The data corresponds to aluminum particles in a silicone matrix $\left(k_{1}=0.2 \mathrm{~W} / \mathrm{m} . \mathrm{K}\right)$. Note that the latter three models are constrained to the sphere limit of $\phi=0.527$. It is remarkable to notice that the Zehner and Schlunder model (1970) begins to depart from Maxwell and Rayleigh model as early as $\phi=0.1$, even though the latter models are known to be accurate up to about $\phi \approx 0.25$. It is seen that the modified Rayleigh model due to Meredith and Tobias (1915) is an improvement over Rayleigh and Maxwell models for $\phi>0.3$. The discrepancy between Zehner and Schlunder model and those of Rayleigh and Maxwell increases with an increase in $\phi$. The Woodside model exceeds that of Zehner and Schlunder (1970). Good agreement is obtained between the Zehner-Schlunder model and the data covering a range up to $\phi=0.6$. The results clearly establish the improvement of Zehner-Schlunder model in representing the data.

Figs. 4a-d describe a comparison of the predictions of Zehner-Schlunder (1970) with the test data for various values of $k_{2} / k_{1}$ as obtained by various investigators. Fig. 4a compares the theory with the data of Wong and Bollampally (1999) for $k_{2} / k_{1}=7.7$ up to $\phi \approx 0.5$. The data are obtained for spherical silica phenolic inclusions embedded in epoxy resin $\left(k_{1}=0.195 \mathrm{~W} / \mathrm{m} . \mathrm{K}\right)$. Excellent agreement is noted between the theory and the data.

A comparison of the theory with the data of Wong and Bollampally (1999) for $k_{2} / k_{1}=184.6$ is sketched in Fig. 4b. The data correspond to spherical aluminum particles in an epoxy resin ( $\left.k_{1}=0.195 \mathrm{~W} / \mathrm{m} . \mathrm{K}\right)$. The data span up to $\phi=0.5$, and satisfactory agreement is seen between the theory and the data.

Figure $4 \mathrm{c}$ indicates a comparison of the theory with the data of Woodside and Mesmer (1961) for $k_{2} / k_{1}=325.8$. The data are obtained up to $\phi=0.8$, and correspond to quartz sand particles in air. Excellent agreement between the prediction and the measurements is noticed over the entire range of solid fraction.

In Fig. 4d a comparison of the prediction with the data of Lin et al. (1993) for $k_{2} / k_{1}=$ 1067.9 and the data of Wong and Bollampally (1999) for $k_{2} / k_{1}=1128.2$. The data for $k_{2} / k_{1}=$ 1128.2 correspond to SCAN particles in epoxy resin, while the data of $k_{2} / k_{1}=1067.9$ correspond to cupric oxide in epoxy resin. The value of $k_{2} / k_{1}$ differs by 5.3 percent in these two sets of measurements. It is observed that the data for the effective conductivity are consistent with each other considering the closeness of the parameter $k_{2} / k_{1}$. Here again the theory satisfactorily describes the data.

The results of Figs. 3 and 4 for the effective thermal conductivity are represented in Fig. 5 as a function of $k_{2} / k_{1}$ with $\phi$ as a parameter. While Fig. 1shows comparisons restricted to a narrow range of solids fraction near $\phi=0.6(\psi=0.4)$, Fig. 3 demonstrates a comparison for a wider range of solids fraction. The satisfactory performance of Zehner-Schlunder model is thus evident. 


\section{Discussion}

All the theoretical models referred to in this work are point contact models. In all the measurements considered here for comparison purposes, there is unfortunately no information reported as to how the effect of weight or other forces that could increase the contact area are taken into account.

The preceding comparisons suggest that the Zehner-Schlunder model appears to be promising for estimating the effective thermal conductivity of water frost for which the thermal conductivity ratio (ice to air) is about 100 . However in frost growth and densification in addition to thermal conduction (stagnant), additional physical processes such as mass diffusion (of vapor) and perhaps eddy convection become important. A detailed investigation of the frost thermal conductivity prediction building on the Zehner-Schlunder model and validation with measurements is currently undertaken by the author, and is beyond the scope of the present work.

\section{Conclusions}

Comparisons of the Zehner-Schlunder model for the effective thermal conductivity of porous media containing spherical particles with the experimental data suggest that the model is satisfactory over a broad range of solid-to-fluid thermal conductivity ratio as high as 1200 . The validity of the model is also confirmed over a broad range of solids fraction as high as 0.8 in certain case where data are available. It is remarkable that the one-dimensional representation of this model is able to describe the effective conductivity of porous media over such an extended range of parameters. Considering these comparisons, the model appears to be promising in the description of the effective conductivity of water frost, for which the solid-to-fluid (ice-to-air) thermal conductivity ratio is about 100 and is well within the acceptable range of the model.

\section{Acknowledgments}

The author is grateful to the referees for helpful suggestions in improving the manuscript. 


\section{References}

Bruggeman, D.A.G., Calculation of various physical constants in heterogeneous substances. I. Dielectric constants and conductivity of composites from isotropic substances (German), Ann. Phys., Vol. 24, pp. 636-679, 1935.

Cheng, P., and Hsu, C-T, The effective stagnant thermal conductivity of porous media with periodic structures, Journal of Porous Media, Vol. 2, pp. 19-38, 1999.

Hertz, H., J. Math. (Crelle's Journal), Vol. 92, 1881.

Hsu, C.T., Cheng, P., and Wong, K.W., Modified Zehner-Schlunder models for stagnant thermal conductivity of porous media, Int. J. Heat and Mass Transfer, Vol. 37, pp. 2751-2759, 1994.

Kandula, M., On the effective thermal conductivity of water frost considering mass diffusion and eddy conductivity, submitted to Int. J. Heat mass transfer, October 2009.

Kanuparthi, S., Subbarayan, G., and Siegmund, T., An efficient network model for determining the effective thermal conductivity of particulate thermal interface materials, IEEE Transactions on Components and Electronic packaging, Vol. 31, pp.611-621, 2008.

Kaviany, M., Principles of Heat Transfer in Porous Media, New York, Springer-Verlag, New York, 1995, pp. 143-148.

Lin, F., Bhatia, G.S., and Ford, J.D., Thermal conductivities of powder-filled epoxy resins, J. Appl. Polymer Sci., Vol. 49, pp. 1901-1908, 1993.

Maxwell, J.C., A Treatise on Electricity and Magnetism, Clarendon Press, Oxford, 1873, p. 365.

Meredith, R.E., and Tobias, C.W. , Resistance to potential flow through a cubical array of spheres, J. Applied Phys., Vol. 31,pp. 1270-1273, 1969.

Nozad, S., Carbonell, R.G., and Whitaker, S., Heat conduction in multiphase systems, I: Theory and experiments for two-phase systems, Chem. Eng. Sci., Vol. 40, pp. 843-855, 1985.

Rayleigh, Lord, On the influence of obstacles in rectangular order upon the properties of a medium, Philosophical magazine, Vol. 56, pp. 481-502, 1892.

Runge, I., Zur electrischen Leitfahigkeit metallischer Aggregate, Z. Tech. Physik, Vol. 6, pp. 61$68,1925$.

Tien, C.L., and Vafai, K., Statistical bounds for the effective thermal conductivity of microsphere and fibrous insulation, AIAA Progress Series, Vol. 65, pp. 135-148, 1979 (see also AIAA Paper No. 78-874). 
Timoshenko, S.P., and Goodier, J.N., Theory of Elasticity, 3rd. ed., New York, McGraw-Hill Book Co., New York, 1970.

Wong, C.P., and Bollampally, R.S., Thermal conductivity, elastic modulus, and coefficient of thermal expansion of polymer composites filled with ceramic particles for electronic packaging, J. Appl. Polymer Sci., 74, pp. 3396-3403, 1999.

Woodside, W., Calculation of the thermal conductivity of porous media, Can. J. Physics, Vol. 36, pp. 815-823, 1958.

Woodside, W., and Messmer, J.H., Thermal conductivity of porous media. I. Unconsolidated sand, J. of Appl. Physics, 12, No. 9, pp. 1688-1699, 1961.

Zehner, P., and Schlunder, E.U., Thermal conductivity of granular materials at moderate temperatures (in German), Chemie Ingr. Tech., 42, pp. 933-941, 1970. 


\section{List of Figures}

Figure 1. Comparison of predictions from Zehner and Schlunder model (1970) with the data of Nozad et al. (1985).

Figure 2. Comparison of various models for the full spectrum of porosity.

Figure 3. Comparison of Zehner and Schlunder model (1970) and other models with the data of Kanuparthi et al. (2008).

Figure 4. Comparison of Zehner and Schlunder model (1970) with the data for a range of thermal conductivity ratio from 8 to 1100 .

Figure 5. Comparison of Zehner and Schlunder model (1970) with the data with the solids fraction as a parameter. 


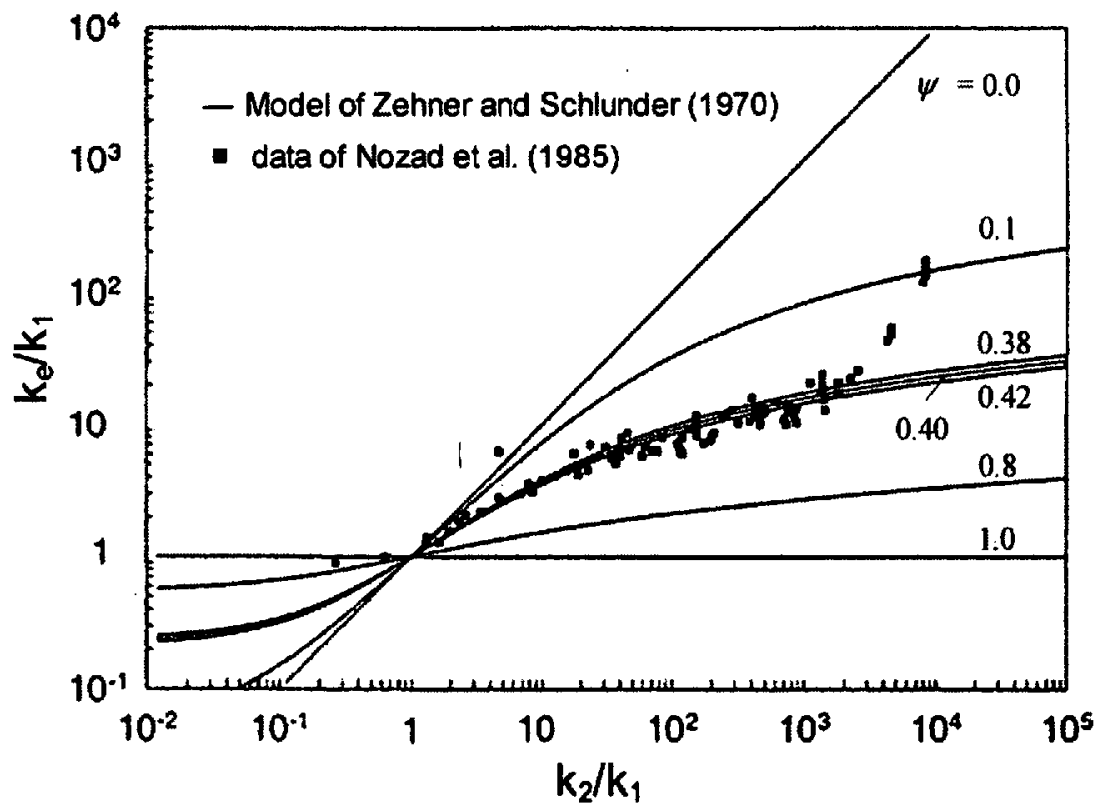

Figure 1. Comparison of predictions from Zehner and Schlunder model (1970) with the data of Nozad et al. (1985).

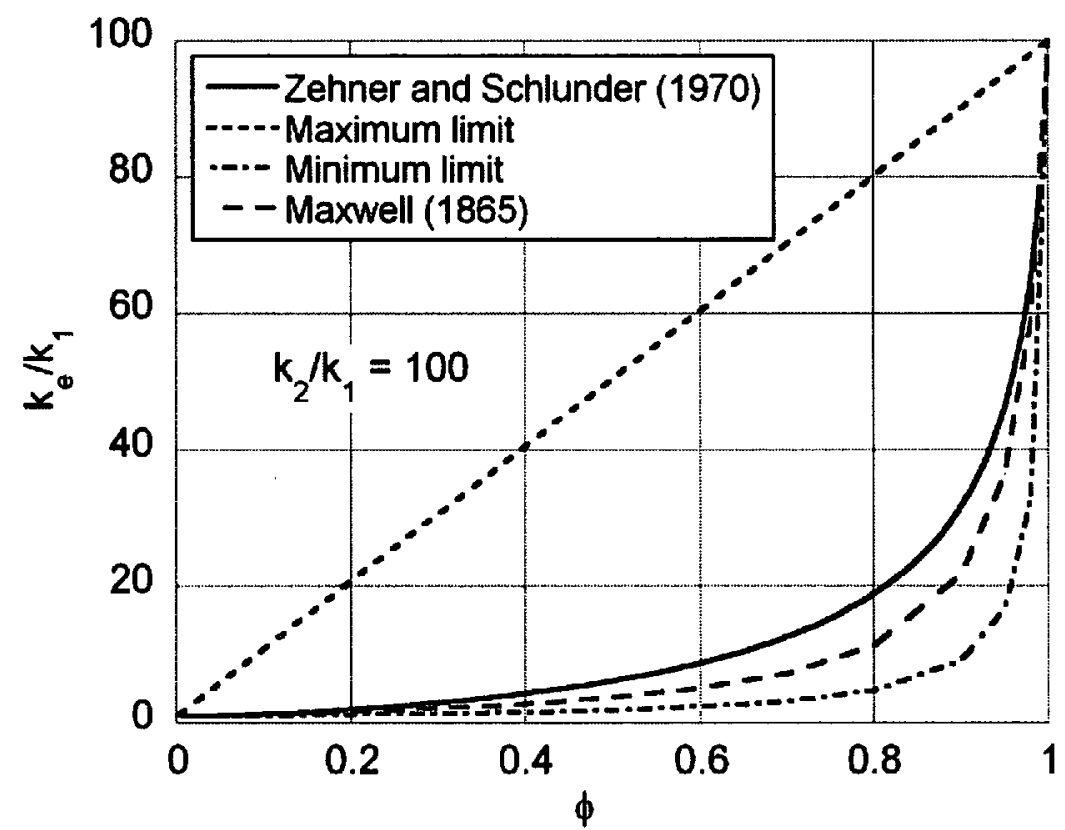

Figure 2. Comparison of various models for the full spectrum of porosity. 


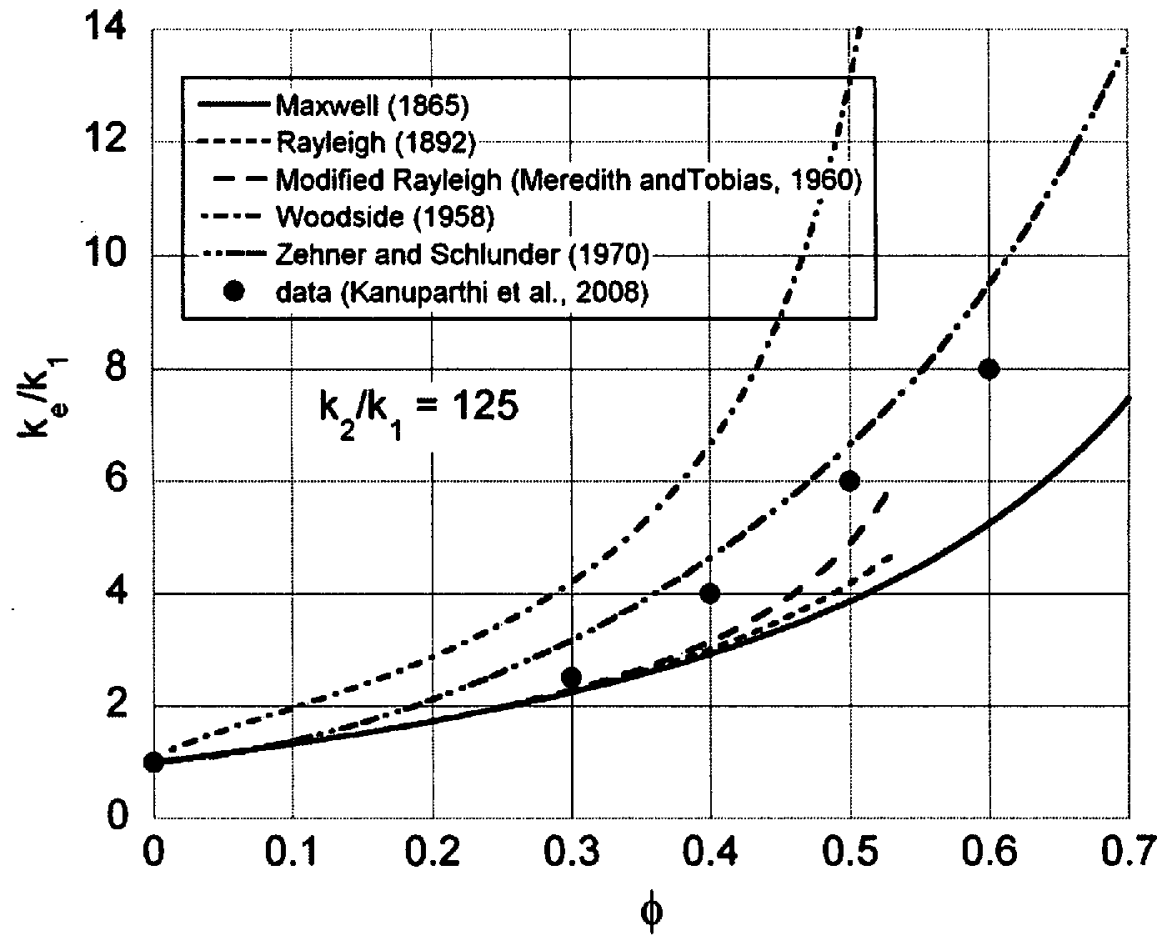

Figure 3. Comparison of Zehner and Schlunder model (1970) and other models with the data of Kanuparthi et al. (2008). 


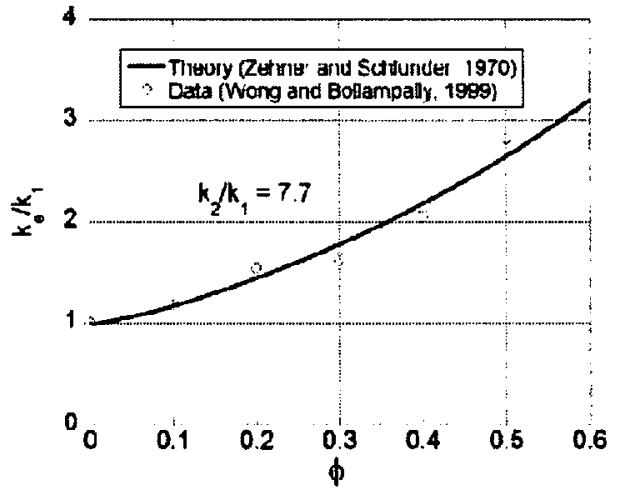

(a)

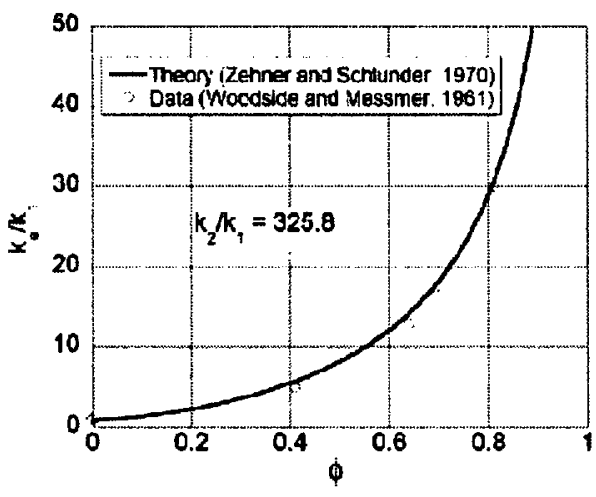

(c)

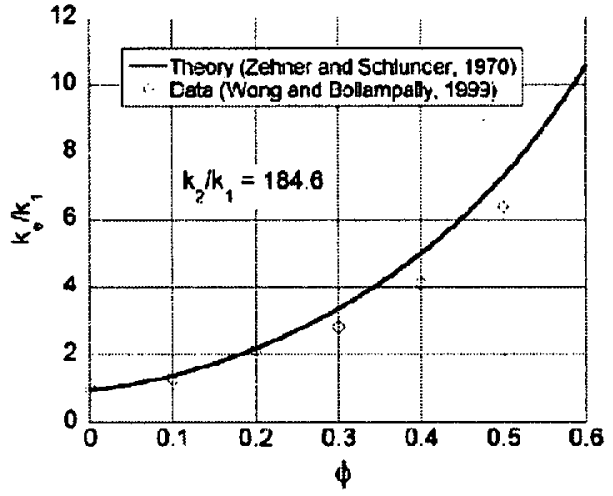

(b)

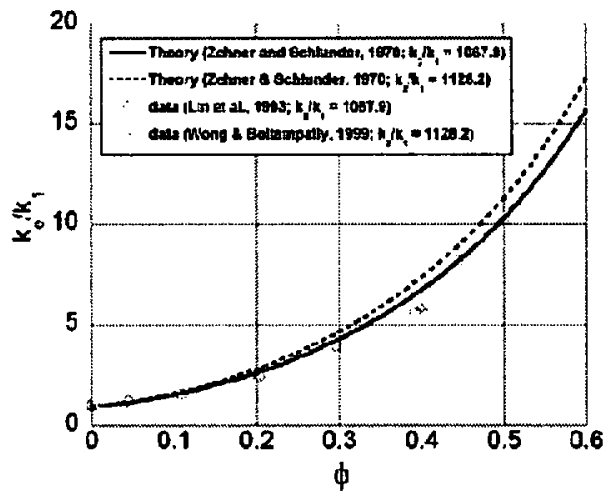

(d)

Figure 4. Comparison of Zehner and Schlunder model (1970) with the data for a range of thermal conductivity ratio from 8 to 1100 .

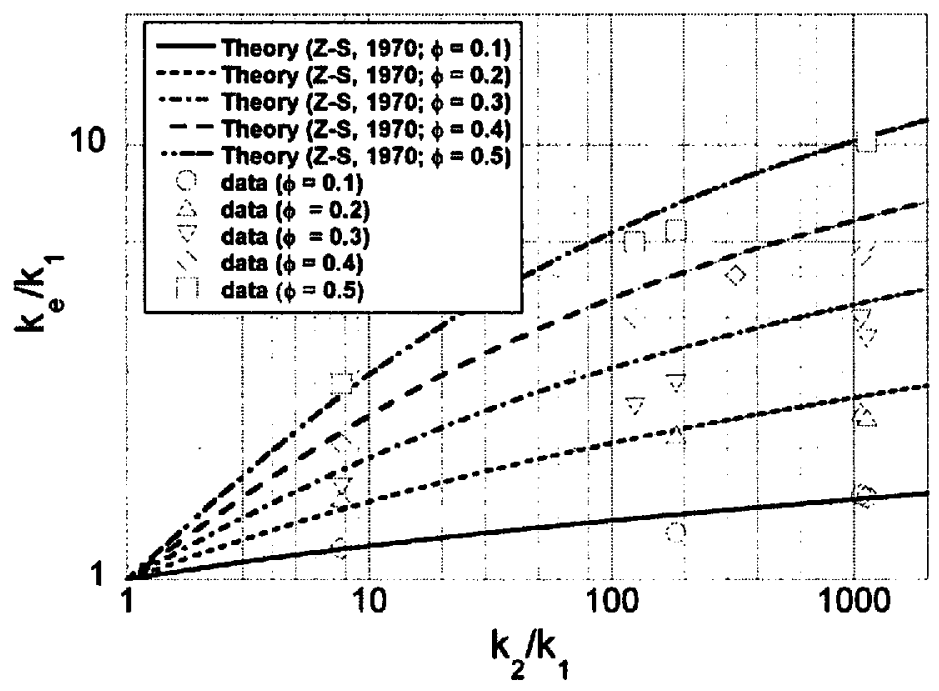

Figure 5. Comparison of Zehner and Schlunder model (1970) with the data with the solids fraction as a parameter. 